\title{
Simplified Model for Reactive Absorption Processes
}

\author{
Prashant Patil and Megan Jobson \\ Department of Process Integration, UMIST, P O Box 88, Manchester, M60 1QD, UK
}

\begin{abstract}
Reactive absorption is an industrially important process. Although much work has been carried out on rigorous modelling of the reactive absorption column (e.g. Kenig et al., 1999), little work has focussed on design and synthesis of reactive absorption processes. Rigorous models are capable of accurately representing process behavior, but they are not suitable for design and synthesis purposes, as they are highly complex and difficult to optimize. Instead, a suitable model, which is simple and capable of accommodating complex structural options such as multiple feeds, intermediate heating or cooling, etc., is required. Shortcut or simplified models are available for physical absorption (Edmister, 1957), but no shortcut model is available for absorption accompanied by reaction.

A new simplified model is developed for reactive absorption. In this work, components are grouped into two sets - the 'key' components, for which there are significant deviations from phase and/or reaction equilibrium, and the 'non-key' components, which essentially achieve equilibrium on each stage. In particular, some of the non-condensable gases present in the gas feed and the non-volatile liquid components may effectively reach equilibrium on each theoretical stage. The key components are related to equilibrium composition by the Murphree stage efficiency. The enhancement factor used in the component efficiency calculation of key components accounts for the effect of chemical reaction on mass transfer in the liquid phase. The non-key components are assumed to be in equilibrium. The Kremser group method (e.g. Seader and Henley, 1998) is used to model the behavior of non-key components. In the simplified model, a simple column is treated as a countercurrent cascade (or block) of separation stages. Only for the top and bottom stages of the block are the stage temperatures and flow rates and equilibrium constants (K-values) calculated rigorously. The behaviour of the whole column section is then modelled by assuming profiles (of temperature, K-values, flow rates, etc.) and applying a modified form of the Kremser equation.

The model developed for a group of stages can represent simple columns with a single vapour feed, a single liquid feed, a single vapour outlet stream and a single liquid outlet stream. For design and synthesis studies, more complex column structures, including multiple feeds, reboilers, condensers, side draws and intermediate heating or cooling, must be accommodated. For complex columns, the simplified model for a group of separation stages is treated as block that is linked to other blocks by interfacing stages, for which structural changes, such as a feed or side draw, are modelled. Rigorous calculations are
\end{abstract}


carried out for the interfacing stages; the blocks between them are represented by the simplified model. The equations mass and energy balances and efficiency relations are solved simultaneously using the Newton-Raphson method.

For the process of gas sweetening, the model has been validated against published plant data (MacKenzie, 1987) and rigorous simulation results, using the Amines property package within HYSYS. The predicted removal of $\mathrm{H}_{2} \mathrm{~S}$ and $\mathrm{CO}_{2}$ from a gas mixture using MDEA in aqueous solution was in good agreement with measured plant data. Both the multiple-feed absorption columns and the solvent recovery column, essentially a distillation column operating at a relatively low pressure, are satisfactorily modelled by the new simplified model.

Keywords: Reactive absorption, Kremser group method, simplified model

\section{References:}

Kenig, E.Y., Schneider, R., Gorak, A., 1999, Rigorous dynamic modelling of complex reactive absorption processes, Chem. Eng. Sci. 54 (21), 5195-5203.

Edmister, W.C., 1957, Absorption and stripping factor functions for distillation calculation by manual- and digital-computer methods, AIChE J., 3 (2), 165-171.

Seader, J. D. and Henley, E. J., 1998, Separation Process Principles, New York, Chichester:Wiley.

MacKenzie, D. H., Prambil, F. C., Daniels, C. A., and Bullin, J. A., 1987, Energy Progress, 7, 31-36. 